\title{
A Beam Profile Monitor for Small Electron Beams
}

\author{
J. Norem* \\ High Energy Physics Division, Argonne National Laboratory \\ Argonne, IL, 60439, USA \\ January 23,1991 \\ ANL-HEP-TR- -91-08 \\ DE91 018595
}

SEP 201991

\begin{abstract}
Measurement of beam properties at the foci of high energy linacs is difficult due to the small size of the waists in proposed and existing accelerators ( $1 \mathrm{~nm}$ - $2 \mu$ ). This paper considers the use of bremsstrahlung radiation from thin foils to measure the size and phase space density these beams using nonimaging optics. The components of the system are described, and the ultimate resolution, evaluated theoretically for the case of the Final Focus Test Beam at Stanford Linear Accelerator Center, is a few nm.
\end{abstract}

\section{Introduction}

The design of efficient linear colliders requires very small beams to produce high luminosities [1]. The small beams, in turn, require high precision and stability in all accelerator components. Producing, monitoring and maintaining beams of the required quality has been, and will continue to be, difficult. This paper describes a beam monitoring system which could be uscd to measure beam size and stability at the final focus of a beamline or collider.

The system consists of a Bremsstrahlung radiator at the focus of the electron beam, a single sided collimator to produce a bremsstrahlung shadow, and a slit and detector system to measure the shape of the shadow edge.[2] In addition, sweeping magnets and shielding are required to disperse and absorb electron and photon backgrounds, Fig.

-Work supported by the U. S. Department of Energy, Division of Bigh Energy Physics, Contract W-31-109-ENG-38. 
1. The linear dimensions are not critical. The sharpness of the shadow is inversely proportional to the size of the spot at the bremsstrahlung source. The optics of the system can be shown by plotting beam phase space at the focus, at tise collimator and at the detector downstream, as shown in Fig. 2. By moving the detector slit back and forth it is possible to measure the shadow width, and hence the beam profile. Moving the primary collimaior alters the initial $x^{\prime}$ values, thus the whole phase space density distribution, $\rho\left(x, x^{\prime}, y, y^{\prime}, t\right)$, can be measured. At the final focus of the FFTB [3] or the JLC/ATF[4] the technique should give a resolution of a few nm and less than I psec, though the best resolution in time and space may be incompatible. The parameters of the proposed beamlines are shown in Table I.

The technique starts with the following advantages over other systems: enormous signal brightness, (bremsstrahlung intensity / emittance), simple analysis, (using theory developed in 1815[5]), and the detectors are, in principle, blind to the most troublesome background.[6] Implementation is complicated by the fact that the system must operate in or near the electron beam dump and in the presence of showers caused by collimators. It is assumed that a detailed design, including shielding, could be done after the rest of the system was specified.

\section{Components}

Collimators can be made from commercially available metal mirrors, which are described in catalogues with a flatness of $<\lambda / 40 \sim 14 \mathrm{~nm}$, and surface roughness of better than 1nm.[7] Six invar mirrors with dimensions of $\sim 5-10 \mathrm{~cm}$, optically polished to a few nm surface roughness, would cost about $\$ 3000$, and take about 10 weeks to fabricate.[8] The ideal shape of the primary collimator would be slightly convex with the front edge tangent to one side of the slit and the downstream edge tangent to the other side of the slit. With a $1 \mu$ slit opening, this would imply a sagitta of about $1 \mathrm{~nm}$, which is essentially flat. It is assumed that guard collimators would be larger, heavier and perhaps water cooled, rough positioning ( $\pm 100 \mathrm{~nm}$ ) of these should be sufficient.

Rough positioning of guard collimators can be done with a number of commercially available systems, such the the Nanomover sold by Melles Griot, which can set $20 \mathrm{~kg}$ loads with $\pm 100 \mathrm{~nm}$ resolution over $25 \mathrm{~mm}$. [7] The primary collimator and final slit would have to be more carefully positioned, possibly to tolerances of $\pm 1 \mathrm{~nm}$. Fine adjustment can be done with piezoactuators or Inchworm drives, sold by Burleigh, for 
motions of $0.1 \mathrm{~nm}$ to $10 \mu$ or larger.[9] It is assumed that the collimators will each be controlled with three actuators, and the precision adjustment of these would be done in real time. Mirrors can be mounted to the structure in a number of ways, a compliant mount, such as pitch, might be desirable.

To examine the penumbra with high resolution, it is necessary to use a slit just before the convertor. A precollimator is inserted upstream of the open side of the slit to reduce shower backgrounds on it. It is assumed that Xe gas at 1 atm can be used as the Cerenkov radiator. With a refractive index $n=1.00071$, the opening angle of radiation is $2.1^{\circ}$, and the minimum detectable electron energy is $\sim 12 \mathrm{MeV}$. This paper assumes that the pair converter is $3.6 \mathrm{~mm}$ thick and the $\check{C}$ radiator is $2 \mathrm{~cm}$ thick, and the combined width due to pair production / shower dimensions and Cerenkov optics is $\sim 100 \mu$. In this geometry, electrons are efficiently used, as the Cerenkov source is inherently slitlike, and the slit on the streak camera may be unnecessary.

Time resolution within the bunch, using a streak camera, adds the requirement that the maximum amount of light be available for high resolution streak pictures. In the Argonne AATF, streak camera measurements have been done with $3 \cdot 10^{10}$ electrons imaged roughly $1 \mathrm{X}$ on a slit set at about $100 \mu$, using $2 \mathrm{~mm}$ of Xe at atmospheric pressure, giving long, bright streaks.[10] For simple intensity measurements, shorter streaks should be sufficient, which should require $I_{e} \sim 8 \cdot 10^{7}\left[\mathrm{e}^{-} m m_{X e}\right]$, with $I_{e}=$ $n_{e}\left(l / L_{R}\right) \eta\left(\dot{\phi} / \sigma_{x^{\prime}}\right) \Delta z$, where $n_{e}$ is the number of electrons, $l / L_{R}$ is the thickness of the converter in radiation lengths $, L_{R}, \eta$ is the number of electrons detected for one equivalent full energy photon on the detector, which is calculated by monte carlo and is roughly $13, \phi / \sigma_{x^{\prime}}$ is the acceptance of the detector divided by the divergence of the beam, and $\Delta \cdot$ is the length of Cerenkov radiator.

Commercial streak cameras have a resolution of 0.6 - 2ps.[13] The streak camera is required for time resolved images, but almost any ionization detector (film, scintillator, ion chambers) could be useful for profile or stability measurements. Čerenkov radiators are a good way of hardening the detector, which is highly desirable. At the highest resolution, the system will probably not produce enough light for time resolved images.

\section{Limitations}

The ultimate resolution of this system is limited by Fresnel diffraction. If the intensity of diffraction pattern on a screen is given by $F(y)$, the width of the pattern is a function 
of

$$
y=v \sqrt{\frac{b \lambda(a+b)}{2 a}}
$$

which gives the dimensions of diffraction patterns on a screen in terms of $v$, the dimensionless variable used to evaluate the Fresnel integrals, and $a=20, b=2 \cdot \mathrm{m}$, and $\lambda$, the wavelength of the gamma's.[5]. The resolution of the system is determined by incoherently adding the diffraction images produced by the bremsstrahlung spectrum as seen by the detector. The acceptance of the detector has been evaluated by means of a short monte carlo program which generates a bremsstrahlung spectrum, computes pair production and subsequently evaluates multiple scattering.[11] The detected FFTB spectrum, which depends somewhat on position and angle cuts, is shown in Fig. 3. This note defines a resolution function as the derivative of this sum of diffraction images and this is shown in Fig 4., for the $\pm 100 \mu$ slits discussed above. The resolution will be nongaussian due to the photon spectrum. Assuming a gaussian for the equivalent resolution of a monochromatic diffraction image, one finds $\sigma_{y,[n m]} \sim 15 E_{[G e V]}^{-1 / 2}$, however high energy photons contribute most of the resolution.

The beam will self focus in the radiator. Magnetic fields $B \sim \mu_{0} I / 4 \sigma_{x} \sim 50 T$ will produce deflections, $\theta_{f, \text { max }} \sim B l / B \rho=0.0002$, which will be comparable to multiple scattering of the primary beam, $\sigma_{m s}=0.014 / p \sqrt{l / L_{R}}=0.00012$, and smaller than the divergence of the $60 \mathrm{~nm}$ beam $\sigma_{x^{\prime}} \sim 0.0006$. This note assumes $l=0.6 \mathrm{~mm}$, $L_{R}=3.6 \mathrm{~mm}, n_{e}=10^{10}, \sigma_{z}=1 \mathrm{~mm}, \sigma_{x}=0.8 \mu$ and $p=50 \mathrm{GeV} / \mathrm{c}$. The self focusing and multiple scattering can be minimized with a thinner primary radiator, which may be desirable under some circumstances. The angular distribution of bremsstrahlung photons relative to the electron beam direction, is roughly $m_{e} / E_{\gamma}$, where $m_{e}$ and $E_{\gamma}$ are the electron mass and photon energy.[11] Only the lowest energy photons should be appreciably affected. Self focusing, multiple scattering and angular effects primarily introduce diffusion in the $x^{\prime}$ dimension of phase space at the focus (see Fig 2) and these effects can be corrected.

Almost comparable resolution can be obtained for the JLC/ATF Final Focus Test [4] if the primary collimator is moved within $0.5 \mathrm{~m}$ of the final focus. Since the expected spotsize is $30 \mathrm{~nm}$, coming from a $\beta$ of $80 \mu$, resolution must be good. The spectrum of detected photons is roughly flat, thus the average photon energy is a larger fraction of the maximum energy. The target thickness and the beam energy cause problems. The target must be thin to fit within the waist of the beam, but this reduces the intensity of the bremsstrahlung photons. The low energy of this beam, $1.54 \mathrm{GeV}$, permits the electrons to be steered away from the photon beam in a smaller distance, but causes 
large multiple scattering, which will detectably increase both the beam divergence and the beam itself in a $20 \mu$ radiator thickness.

Fully utilizing the theoretical resolution will require that the collimators must be mounted on a vibration free surface.[12] Both active and passive vibration damping systems might be considered. The problem is, however, similar to the vibration sensitivity of the final quads in the line, and should probably be solved the same way. The passive damping used on the final quads before the focus should be sufficient to produce useful measurements. Slow drifts and low frequency oscillations will probably require that measurements be taken with a few pulses and collimator positions be maintained using active corrections.

\section{Backgrounds}

The system should be blind to synchrotron radiation, which is troublesome because it is produced by the beam with very small emittance near the final focus. Although the streak camera detects visible and near UV, direct synchrotron radiation from the beam in magnets, plasmas or the primary radiator would be blocked by the bremsstrahlung radiator and pair converter. The optics of the streak camera can be made light tight. The primary means of discriminating between signal and shower electrons is that the brightness of the signal should be much larger than that of the showers, which can be magnetically dispersed in two dimensions. In addition, timing can be used to separate direct photon paths from indirect shower paths

The environment downstream of the final focus will be inhospitable to precision equipment. Mechanical, opticai and electrical components should be tested to make sure they will function in the high radiation environment.

\section{Conclusions}

This note outlines the components and limitations of a system designed to measure beam profiles for high energy colliders and test beams. The beam layout must minimize shower contamination of the bremsstrahlung signai and vibration of components. 


\section{Acknowledgements}

P. Schoessow of ANL produced EGS4 calculations of showers in slits, and G. Fischer of SLAC has provided information on vibration and surveying techniques.

\section{DISCLAIMER}

This report was prepared as an account of work sponsored by an agency of the United States Government. Neither the United States Goverrment nor any agency thereof, nor any of their employees, makes any warranty, express or implied, or assumes any legal liability or responsibility for the accuracy, completeness, or usefulness of any information, apparatus, product, or process disclosed, or represents that its use would not infringe privately owned rights. Reference herein to any specific commercial product, process, or service by trade name, trademark, manufacturer, or otherwise does not necessarily constitute or imply its endorsement, recommendation, or favoring by the United States Government or any agency thereof. The views and opinions of authors expressed berein do not necessarily state or reflect those of the United States Government or any agency thereof. 


\section{References}

[1] R. B. Palmer, Annu. Rev. Nucl. Part. Sci. 40, 529, ('90)

[2] J. Norem, Proceedings of the 1988 Linear Accelerator Conf., Newport News, Virginia, (1988), $\mathrm{p} 430$

[3] K. Oide, Proc. 1989 Particle Accelerator Conf, Chicago, 1989, p1319

[4] K. Oide, KEK, private communication, (1991)

[5] Jenkins and White, Fundamentals of Optics, Fresnel Diffraction, McGraw-Hill (1957)

[6] J. Buon, Particle Accelerators, 31IV, 1247, (1990)

[7] Melles Griot, Irvine Calif. 92714

[8] R Lowrey, Rockwell Power Systems, Albuquerque NM (private communication)

[9] Burleigh Instruments Inc., Fishers, NY, 14453

[10] J. B. Rosenzweig, P. Schoessow, B. Cole, C. Ho, W. Gai, R. Konecny, S. Mtingwa, J. Norem, M. Rosing, and J. Simpson, Phys. Fluids B 2, 1376, (1990)

[11] H. A. Bethe, in Experimental Nuclear Physics Vol 1, E. Segre, ed., John Wiley and Sons Inc. New York, (1953)

[12] R. E. Ruland, G. E. Fischer, Proceedings of the 2nd International Workshop on Accelerator Alignment, Hamburg, Germany, Sept 10-12, 1995. /bf and Stanford Linear Accelerator Laboratory publication SLAC-PUB-5326 (1990)

[13] Hamamatsu Photonic Systems Corp., Bridgewater, NJ, 08807 


\section{Table I}

Parameters of Test Beams

$\begin{array}{lrr} & \text { SLAC/FFTB } & \text { JLC/ATF } \\ E, \mathrm{GeV} & 50 . & 1.54 \\ n_{e} & 10^{10} & 10^{10} \\ \epsilon_{x}, \mathrm{~m} & 2 \cdot 10^{-10} & 10^{-10} \\ \beta_{x}, \mathrm{~m} & 0.034 & 0.014 \\ \sigma_{x}, \mathrm{~m} & 2.6 \cdot 10^{-6} & 3.6 \cdot 10^{-6} \\ \epsilon_{y}, \mathrm{~m} & 4 \cdot 10^{-11} & 10^{-11} \\ \beta_{y}, \mathrm{~m} & 10^{-4} & 8 . \cdot 10^{-5} \\ \sigma_{y}, \mathrm{~m} & 6.3 \cdot 10^{-8} & 2.9 \cdot 10^{-8} \\ \mathrm{f}, \mathrm{Hz} & 10 & 25\end{array}$


Figures

Figure 1. The components of the monitor. The beam is defined by the primary collymater and measured by moving the final slit transverse to the beam. Dimensions are those assumed for the SLAC/FFTB system and are not critical. Other light sensitive detectors could be used instead of the streak camera.

Figure 2. Photon phase space of the system, not to scale. The collimator produces an edge which is measured downstream. A projection in $\mathrm{x}$ space shows the observed beam shape.

Figure 3. Monte carlo simulation of the photon energy spectrum detected with the Cerenkov radiator for the SLAC/FFTB beam, with $\theta_{e}<2$ and $\left|\Delta y_{e}\right|<100 \mu$.

Figure 4. The resolution function, defined as the derivative of the diffraction intensity at the bremsstrahlung radiator. This curve is the effective shape of a beam at the bremsstrahlung radiator in the limit of a zero width slit at the detector, assuming the dimensions given in Fig 1.

zz.tex, January 23, 1991

9 

Detected Photon Spectrum

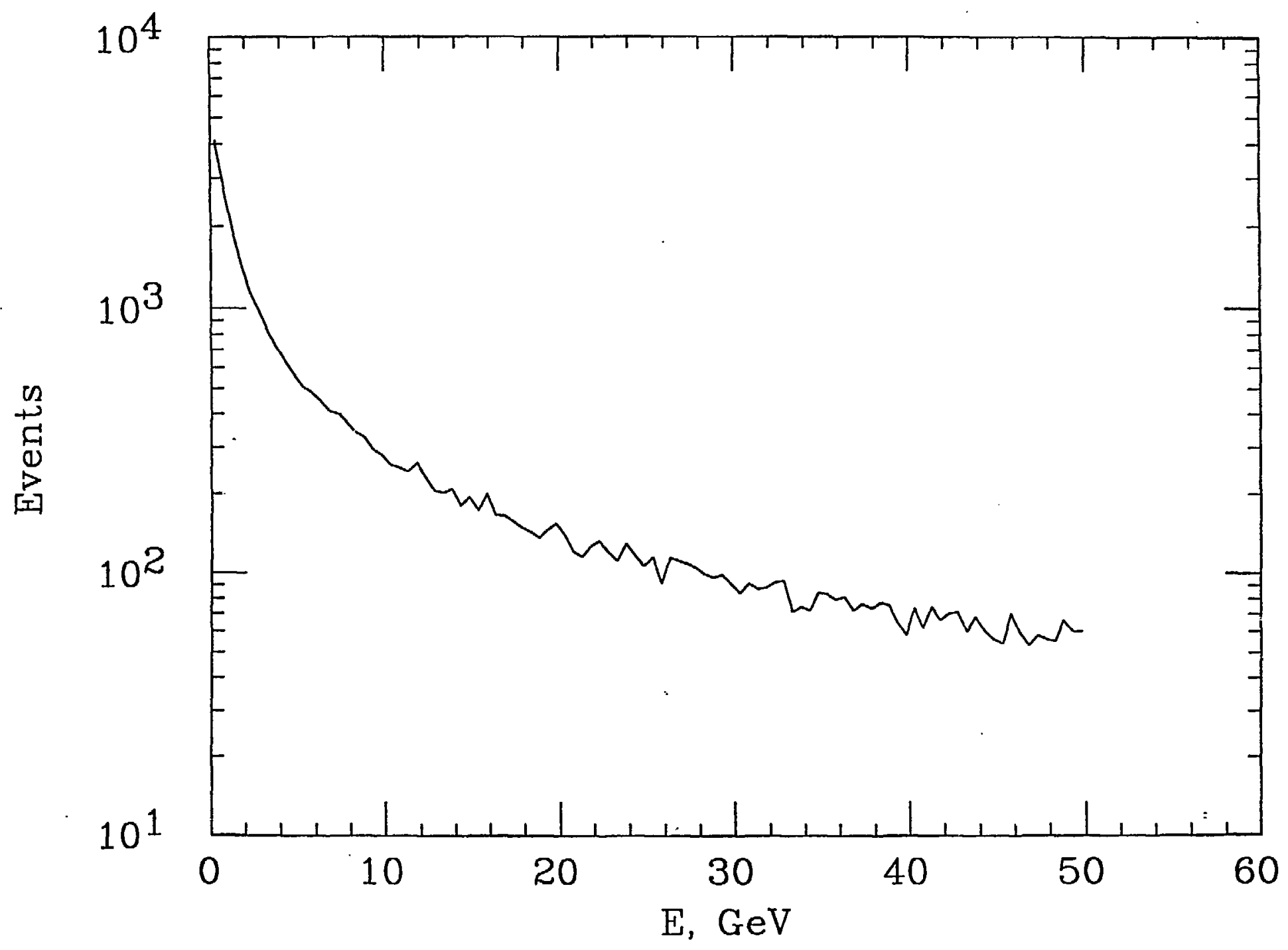


Resolution Function

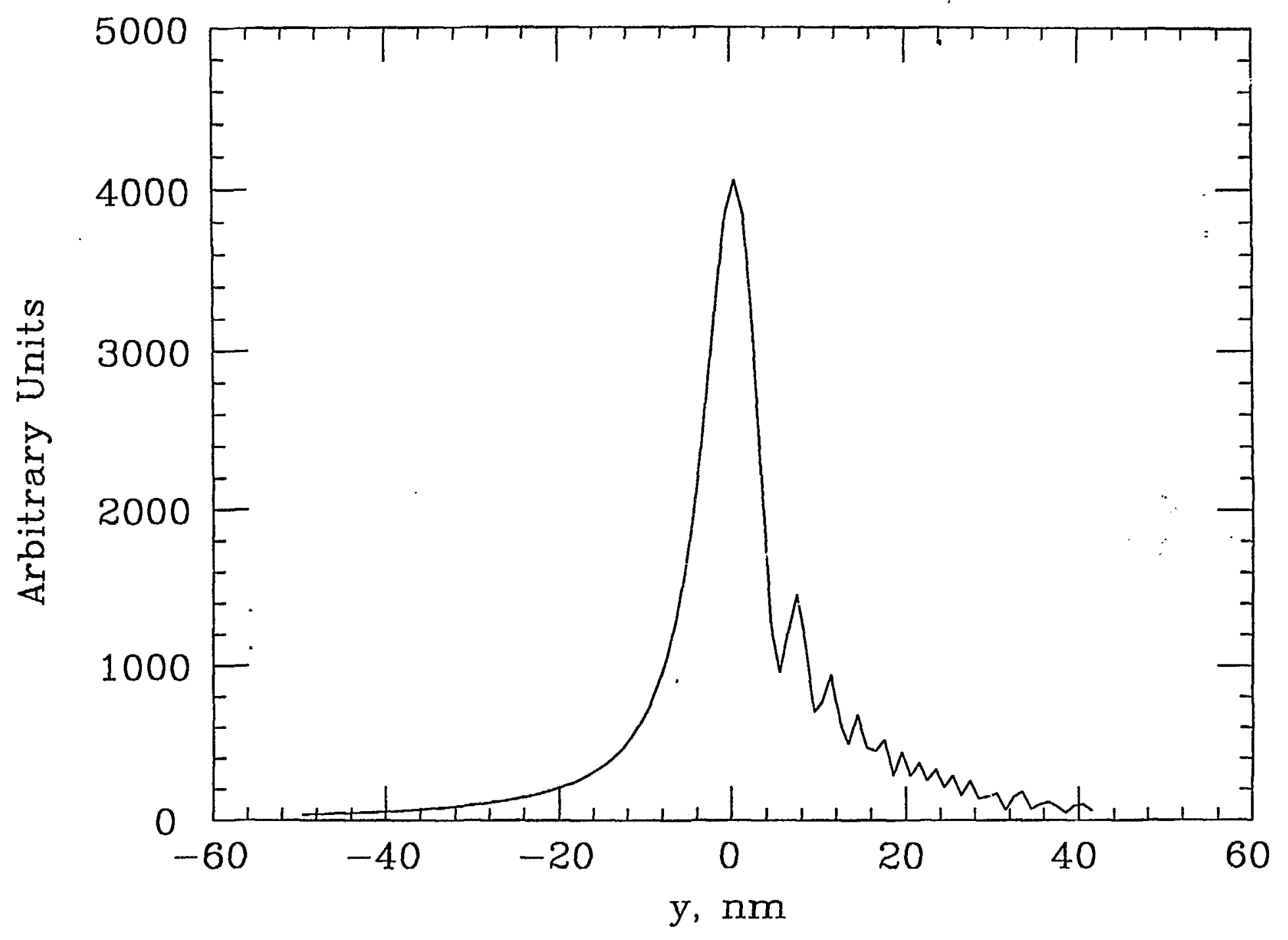

